Revista Ingeniería y Región. 2015;14(2):135-142

\title{
Identificación de las herramientas de la administración de operaciones y producción que se aplican en las empresas transformadoras de material carbonatado del departamento del Huila
}

\section{Identification of the tools of management and production operations that apply in material processors carbonated of Huila}

Reinaldo Alfredo Victoria Bonilla ${ }^{1}$, Diego Iván Caviedes Rubio² y Maykol Andrey Tapia Dussán ${ }^{3}$

\begin{abstract}
Resumen
Este artículo identifica las herramientas de la Administración de Operaciones y Producción (hAOP): administración de producción, investigación de operaciones, seguridad industrial, gestión de talento humano, costos, distribución de planta, mantenimiento industrial, entre otras; que aplican las empresas transformadoras de material carbonatado, en la zona noroccidental del departamento del Huila. El estudio involucró el diagnóstico de un total de 12 empresas de este sector minero, permitiendo reconocer las herramientas y programas que estas implementan, así como las deficiencias en los procesos productivos, el desarrollo y la aplicabilidad de las herramientas o estrategias operativas y productivas. Esta evaluación concederá información adecuada tales como: manejo de inventarios, programación de los planes de mantenimiento, implementación de las ciencias exactas de la ingeniería industrial, favorecerá los procesos, mejorando el manejo de los recursos en benefício social, económico, técnico y político de las empresas de transformación de material minero.
\end{abstract}

Palabras clave: eficacia; calidad; cliente; costos; productividad; proceso; tecnología.

\begin{abstract}
This article identifies the tools of Operations and Production Management (tOPM): production management, research of operations, industrial safety, human talent management, costs, building distribution, industrial maintenance, among others; that use the carbonated materials processing companies, in the northwestern
\end{abstract}

1 Especialista En Seguridad y Prevención de Riesgos Profesionales. Docente Universidad Cooperativa de Colombia. Programa de Ingeniería Industrial. Grupo de Investigación en Administración de Operaciones y Producción ADOPRO, reinaldo.victoria@campusucc.edu.co

2 Magister en Ecología y Gestión de Ecosistemas Estratégicos. Docente Universidad Cooperativa de Colombia. Programa de Ingeniería Industrial. Grupo de Investigación GRIAUCC, caviedesr@campusucc.edu.co

3 Estudiante Ingeniería Industrial Universidad Cooperativa de Colombia, Neiva - Colombia, maikol.tapia@campusucc.edu.co

Recibido: 1 noviembre 2015 - Aceptado: 1 diciembre 2015 
part of the deparment of Huila. The study involved the diagnosis of a total of 12 companies of this mining sector, in order to recognize the tools and programs they implement, as well as deficiencies in production processes, development and applicability of the operating and productive tools or strategies. This evaluation will give appropriate information such as: inventory management, programming of maintenance plans, implementation of sciences of industrial engineering, it will favor processes, improving resource managament in social, economic, technical and political benefit in the mining materials processing companies.

Key words: Efficiency; quality; customer; cost; productivity; process; technology.

\section{Introducción}

La historia de la minería ha presentado períodos de auge y caída, que evidencian una relación equilibrada entre los recursos naturales disponibles, los costos de producción y la capacidad de mantener la rentabilidad. En los últimos años, el liderazgo de la industria minera ha hecho hincapié en que este sector actualmente se ha vuelto más complejo con el advenimiento de las regulaciones más estrictas (Tong, 2014). Se está poniendo mayor énfasis en la importancia de las operaciones sostenibles que se centran en el medio ambiente, integrando efectivamente al entorno social, y los impactos económicos que de la actividad se derivan (Chattopadhyay y Chattopadhyay, 2014). Sin embargo, se podría afirmar que no hay un entorno de competitividad disyuntiva simple y directo, y que no puede haber margen para el logro de resultados ambientales y socioeconómicos más favorables, sin poner en peligro la competitividad del sector industrial a través de diferentes diseños de políticas y estrategias de implementación que incluyan avances o por lo menos actualizaciones en la tecnología con que se desarrollan los procesos (Baptista, 2013; Söderholm et al, 2015).

Las iniciativas de mejora continua en las empresas del sector minero en América Latina son escasamente consideradas (Bebbington et al, 2015). Aunque los ejecutivos de gestión de operaciones se dan cuenta de la importancia de la mejora continua de los procesos, han encontrado que es una tarea difícil, observando que el desafío radica en la creación de una infraestructura estratégica para coordinar proyectos constituidos por secuencias prescritas de pasos para llevar a cabo proyectos y conjuntos de herramientas y técnicas comúnmente utilizadas para ejecutar estos proyectos (Anand et al, 2009; Haslam y Ary Tanimoune, 2016).

El desarrollo de la minería en Colombia fomenta el crecimiento regional y nacional; para el departamento del Huila el sector transformador de material carbonatado se ha convertido en una de sus apuestas productivas (Fuente, 2013), es así que anualmente su producción se ha incrementado en un $10.4 \%$ elevando su participación en el PIB del departamento (Delgado et al, 2015); su mejoramiento debería estar reflejado en una buena administración de sus operaciones y producción, facilitando la búsqueda de soluciones a problemas como: métodos de trabajo, manejo de inventarios, ordenes de pedido, distribución en planta, producción, calidad, seguridad industrial, mantenimiento y gestión ambiental (Sarmiento, 2008); razones por las cuales es necesario determinar qué instrumentos de la (hAOP) aplican las industrias transformadoras de material carbonatado, para así definir planes o estrategias de mejoramiento de todas las operaciones de las empresas del sector (Gonzalez, 2008).

En este estudio se identificaron las (hAOP) que aplican las industrias transformadoras de material carbonatado, considerándose de gran importancia, ya que involucra al sector productivo de la región y genera un conjunto de conocimientos que serán utilizados por futuros investigadores, los cuales darán las soluciones pertinentes a los inconvenientes localizados en los procesos productivos y administrativos de estas organizaciones (Cárdenas, 2008; García, 2008). Para identificar las herramientas, se tuvo en cuenta tanto las decisiones estratégicas y tácticas que se toman en las industrias (porque les permitirá tener control de la proyecciones a corto plazo en la demanda del producto, planear sus recursos, las cuales ayudan a determinar las competencias de las condiciones de estas), como también enfocarse en la aplicación de estándares de operaciones y producción de las industrias del sector y mejorar su desarrollo competitivo (Robilliard, 2005).

\section{Materiales y Métodos}

\section{Área de Estudio}

El estudio tuvo como área de influencia la región noroccidental del departamento del Huila, compren- 
dida por los municipios de Palermo, Tello y Neiva (Figura 1), donde la actividad de las industrias transformadoras de material carbonatado es más significativa. En total se caracterizaron 12 empresas de este subsector de la minería.

\section{Caracterización}

Se diseñó el instrumento de recolección de información adecuado, conforme a los requerimientos que permitan identificar y caracterizar las (hAOP), que aplican las industrias transformadoras de material carbonatado en sus procesos productivos. Este instrumento fue una encuesta que se aplicó a gerentes, los cuales constituyeron la población involucrada en el estudio. La encuesta consta de un total de 72 preguntas dirigidas a evaluar la implementación de técnicas o estrategias como: la ubicación y localización de instalaciones industriales; administración total de la calidad; pronósticos de la demanda; necesidades de capacidad; sistemas de inventarios; procedimientos de dirección de proyectos y subprogramas de seguridad y salud en el trabajo.

Para el diligenciamiento de la encuesta y durante la entrevista se realizó con cada gerente o representante de las empresas una inducción referente a conceptos básicos sobre cada una de las (hAOP) evaluadas desde el contexto en que se desarrolla la actividad productiva y administrativa de las empresas, con el objetivo de evitar sesgos o inconsistencias en la información suministrada. De igual manera, se realizó una serie de visitas de observación directa a cada uno de los procesos productivos de dichas empresas empleando listas de chequeo para verificar algunos datos obtenidos.

\section{Resultados y discusión}

\section{Estrategia de ubicación y localización de instalaciones industriales}

E1 91\% de las industrias transformadoras de material carbonatado utilizaron estrategias (económicas, sociales, ambientales, etc.), para ubicar o situar las instalaciones de sus industrias, con el fin de reducir los costos de producción y tener ventajas competitivas. Por otro lado, con un $45.45 \%$, la técnica que más se utilizó para la localización de las instalaciones de las industrias transformadoras de material minero carbonatado, fue el método de "transporte", ya que consideran que los costos del transporte de la materia

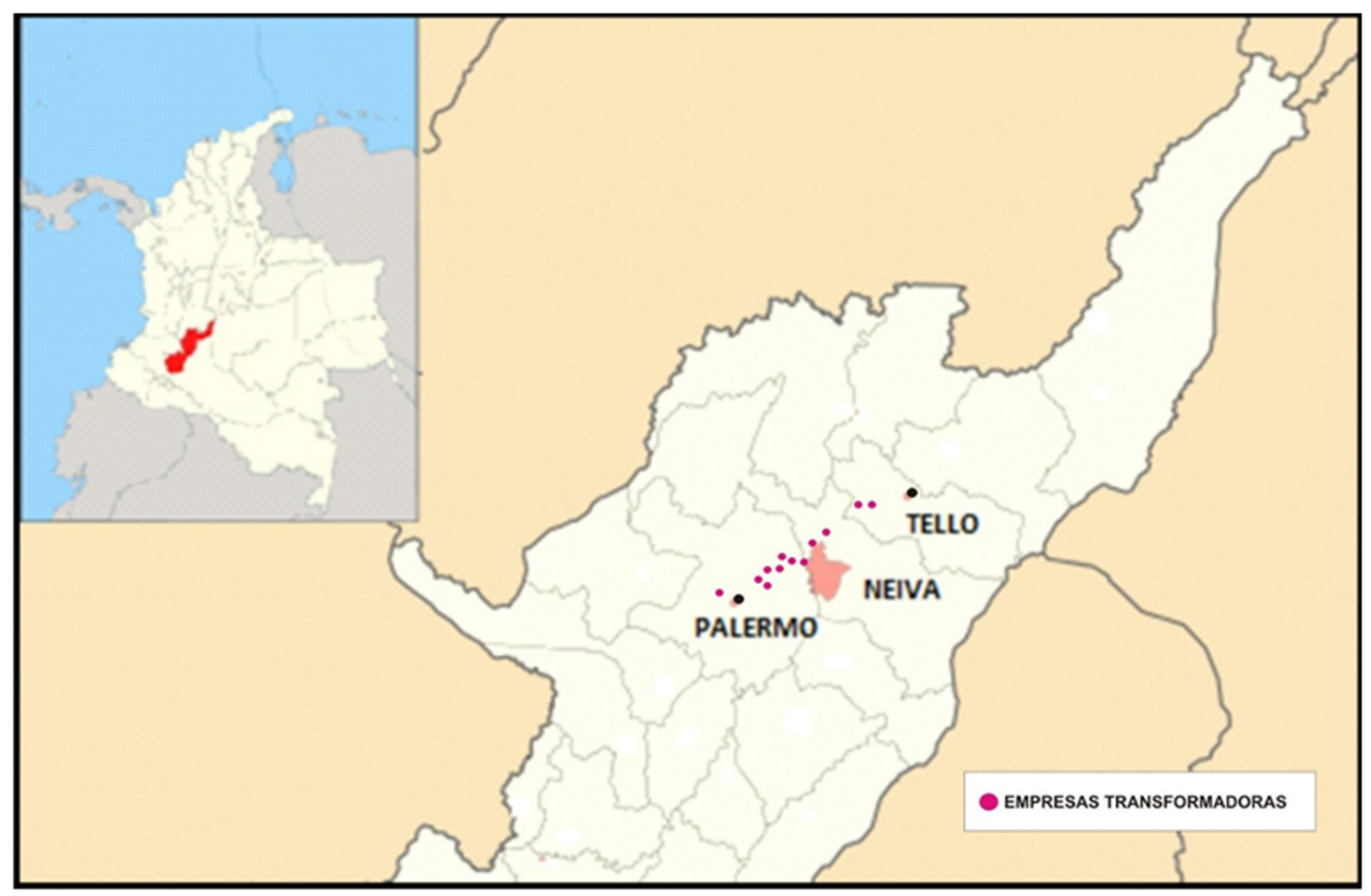

Figura 1. Ubicación de las industrias en la región noroccidental del departamento del Huila. 
prima y del producto terminado, son importantes a la hora de realizar balances del costo-beneficio; los otros métodos más empleados por las empresas objeto de estudio, hacen referencia a los métodos "factores flexibles" y "centroide" con un $27.3 \%$ y un $18.2 \%$ respectivamente. La elección de lugares apropiados para las zonas industriales de este sector, se ha convertido en una decisión compleja, debido a que generalmente presentan diferentes condiciones geográficas, entornos de producción y distancias de las minas. Así mismo, se enfrenta a conflictos de objetivos o intereses entre entidades que pueden tomar o liderar estas decisiones. Una hace referencia al gobierno local, que considera que la ubicación de estas industrias se debe basar en la distribución de la sección de las minas y el medio ambiente regional, a fin de minimizar la contaminación por emisiones y maximizar la eficiencia de las operaciones, y por otro lado, cada empresa elige una zona específica para reducir sus costos totales (Gang et al, 2015).

\section{Administración total de la calidad}

Este tipo de industrias transformadoras en un 46\%, hacen uso de los diagramas de procesos (DPrc), ya que tienen en cuenta todo lo que está inmerso a este para mejorar y controlar la calidad de sus productos. En segundo lugar se identificó a la herramienta "causa-efecto (DC-E)", con un 18\% de utilización entre las empresas evaluadas. Herramientas como los Diagramas de Pareto (DP), Desarrollo de la Función de Calidad (DFC) y el Control Estadístico de Procesos (CEP), se emplean en menor proporción por estas empresas, con solo un $9 \%$ de representatividad (Figura 2).

\section{Pronostico de la demanda}

El tipo de pronóstico más utilizado es el análisis cualitativo con una implementación del $37 \%$ en este sector, este tipo de pronóstico es más exacto y brinda confiabilidad en la previsión de la demanda. La otra herramienta con mayor índice de utilización es el análisis de series de tiempo con un $27 \%$. Sin embargo, se evidencio el desconocimiento de esta herramienta en un $36 \%$ de las empresas evaluadas. Los riesgos de previsión de la demanda existen ampliamente en la economía de las empresas de diferentes industrias. En primer lugar, existe el riesgo de que la empresa sobrestima la demanda y fabrica o produce órdenes de más bienes de los que va a ser capaz de vender. Finalmente, la empresa debe carga con la reducción de beneficios o incluso una pérdida financiera neta. El otro tipo importante de los riesgos es que el negocio subestima la demanda, esto lleva a los niveles de producción insuficiente, dando lugar a escasez y una oportunidad perdida para la empresa (Jian et al, 2015).

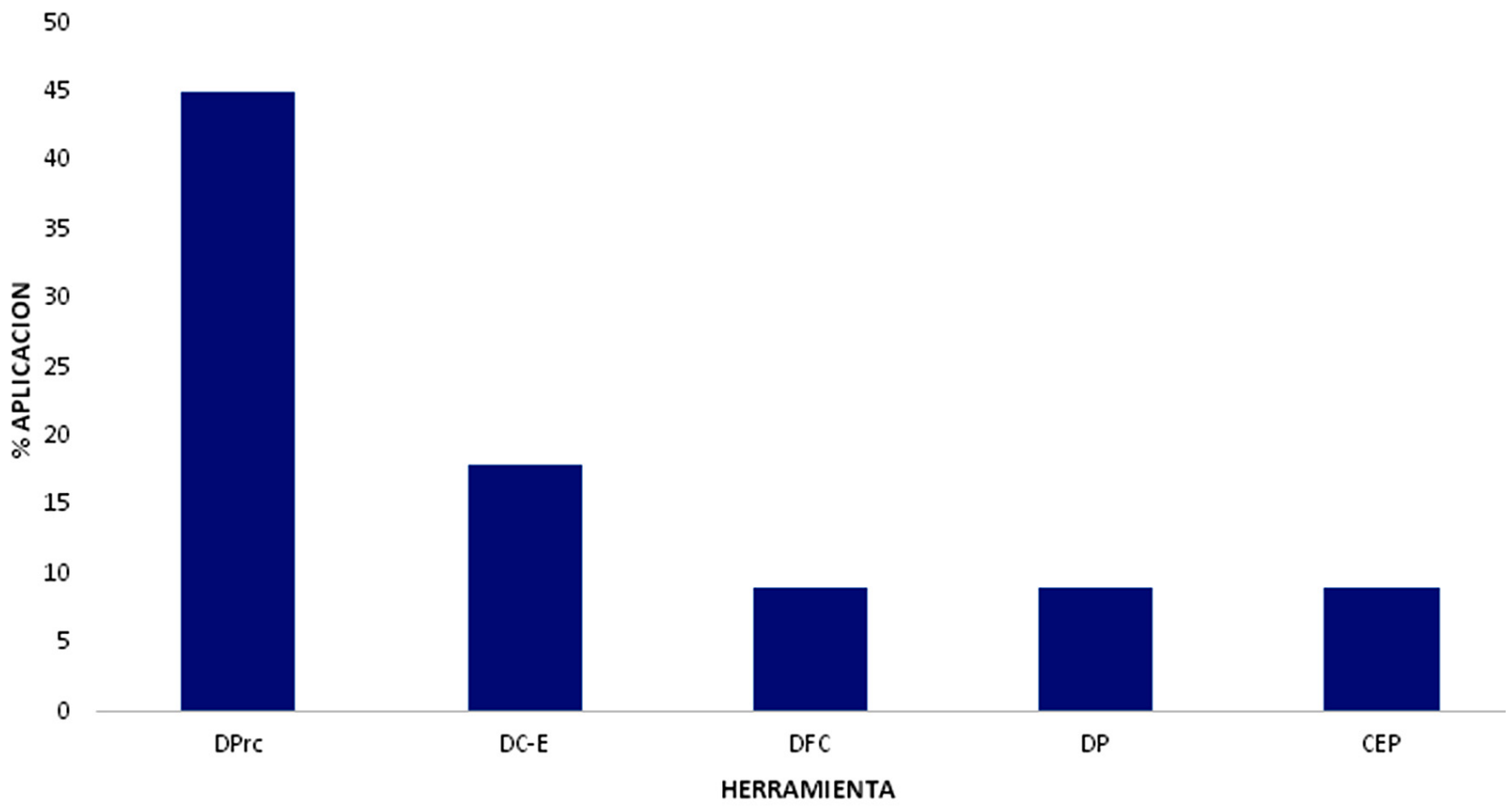

Figura 2. Porcentajes de aplicación de herramientas de la administración total de la calidad. 


\section{Necesidades de capacidad}

E1 91\% de las industrias transformadoras de material minero carbonatado, planifican las necesidades de capacidad, ya que les sirve para conocer los recursos que necesitan para cumplir con las órdenes de pedido y poder cumplir las metas programadas. Si se observa bajo la actual tendencia de negocios de interacción de la cadena de suministros y externalización global, la reducción de plazo de ejecución es esencial para optimizar la eficiencia global de la cadena de suministro. Una parte importante del tiempo de espera es el tiempo de procesamiento de pedidos, durante el cual el proveedor comprueba la viabilidad de la capacidad de la instalación para la producción de un pedido a tiempo; lo que implicaría tiempos de procesamiento más cortos, reduciendo tiempos de ventaja en la generación y el inventario, y el aumento de la eficiencia y la competitividad de la cadena de suministro, siempre que está presente una fuerte integración logística (Olhager y Prajogo, 2012; Hung et al, 2013).

\section{Sistemas de inventarios}

Se observa que el sistema de inventario más utilizado por esta industria es el modelo de seguridad (36\%) (Figura 3), debido a que este vigila constantemente el nivel de inventario y hace un pedido nuevo cuando las existencias alcanzan cierto nivel, además como la demanda es variable se hace necesario un inventario de seguridad para obtener un cierto nivel de protección contra las existencias agotadas. Sin embargo, un porcentaje igual de las empresas incluidas en el estudio no presentan o aplican ningún sistema de inventario. Un $18 \%$ en iguales proporciones manejan los sistemas de inventario por periodo fijo y periodo único y otro $9 \%$ de las empresas transformadoras evaluadas en este estudio desconocen la existencia de sistemas de inventarios.

\section{Procedimientos de Dirección de Proyectos}

Se determinó que el $46 \%$ de las industrias transformadoras de material minero carbonatado implementan los diagramas de Gantt para los procedimientos de dirección de proyectos, porque son herramientas de bajo costo y les permite asegurar que todas las actividades estén planificadas, su orden de realización y por ende la duración global del proyecto. Otro $27 \%$ emplean los diagramas PERT-Gantt y el $9.09 \%$ de las empresas utilizan los diagramas PERT (Programa de Evaluación y Revisión Técnica) para establecer la ruta crítica y así llevar un mejor control de los proyectos que se adelantan en la industria. Por otra parte se pudo afirmar que aunque algunas empresas planifiquen sus proyectos no están administrándolos, que el 19\% de las empresas dicen no utilizar ningún método de dirección de proyectos (Figura 4), queriendo decir que no controlan los recursos, tiempos de ejecución de actividades y el tiempo total del proyecto.

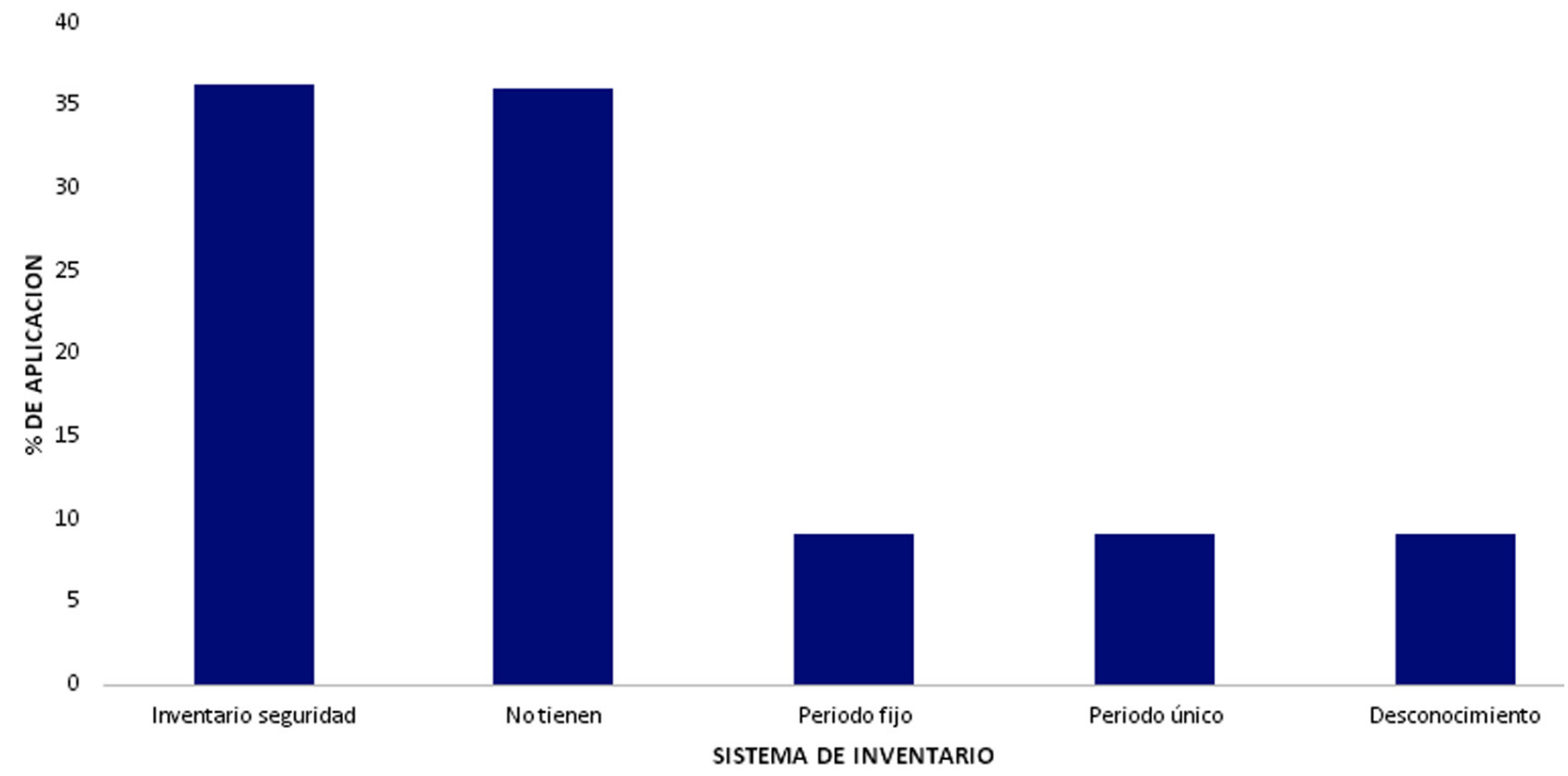

Figura 3. Porcentajes de aplicación de Sistemas de Inventarios en la industria transformadora de material calcáreo en el departamento del Huila. 


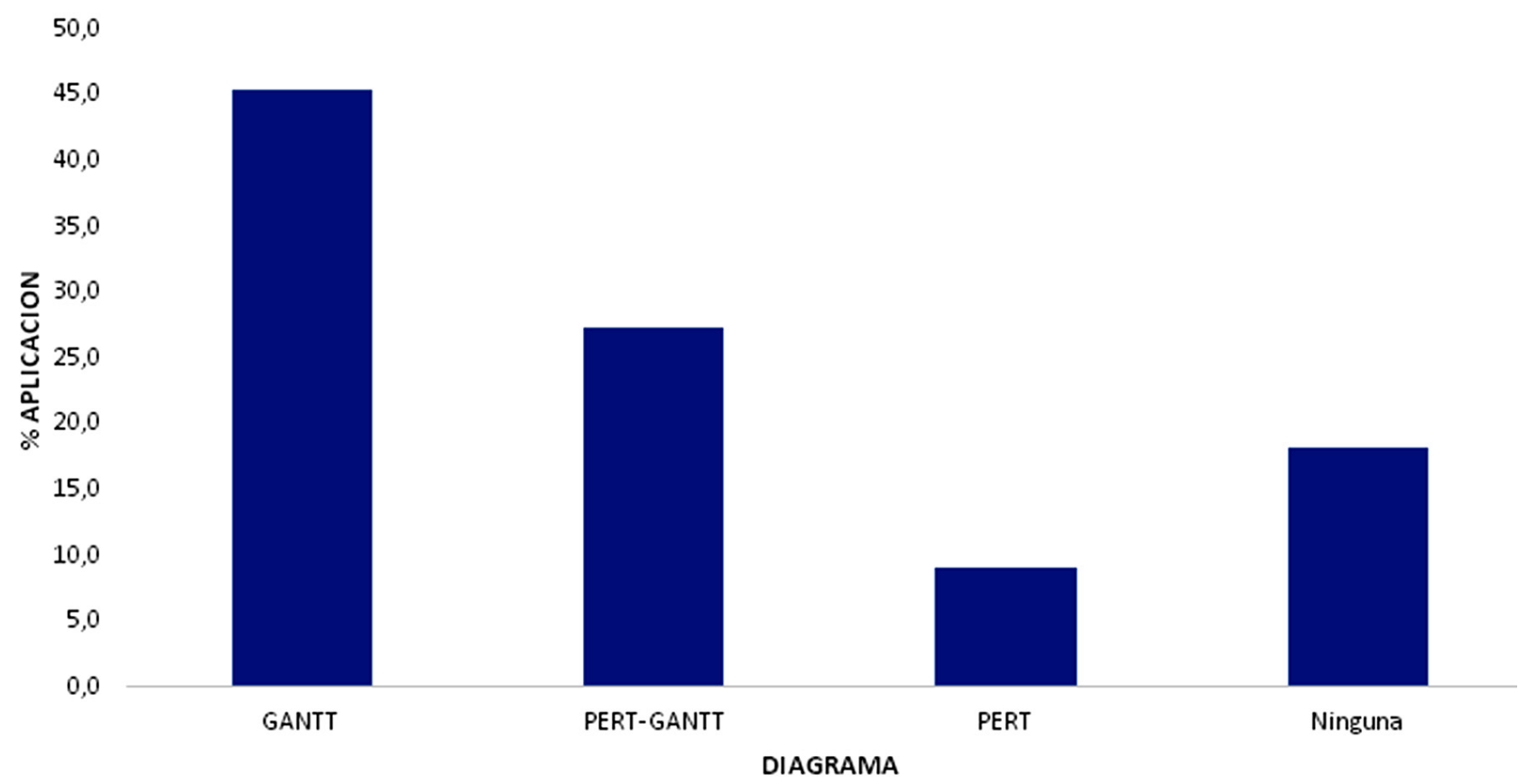

Figura 4. Porcentajes de aplicación de diagramas como procedimientos de Dirección de Proyectos.

\section{Subprogramas de Seguridad y Salud en el Trabajo}

Se evidencia que el $46 \%$ de estas industrias transformadoras implementan en subprograma de medicina preventiva, un 36\% implementa el subprograma de medicina del trabajo y un $18 \%$ aplicó el subprograma de higiene y seguridad industrial; considerándose un alto porcentaje de incumplimiento en la implementación de los subprogramas de salud ocupacional y seguridad industrial. Aunque la necesidad de proporcionar un ambiente de trabajo seguro y saludable para los empleados ha sido durante mucho tiempo la responsabilidad de la administración y de la empresa en su conjunto (Amponsah et al, 2015), por ello, una oportuna y bien dirigida vigilancia por parte de las entidades gubernamentales y las sanciones que se pueden presentar en este sector, podrían evitar incrementos en la tasa de accidentalidad y evidenciar un incremento de la productividad, debido al aumento en la gestión del empleado (Gowrisankaran et al, 2015). En este sector los niveles de ruido y de polvo respirable son altos, con muchas actividades dentro del proceso de producción que exponen directamente al trabajador con la fuente de emisiones y los esfuerzos para mejorar la tasa de emisiones de Solidos Suspendidos Totales (SST), para responder al problema, el sector se ve limitado por la falta de formación y la coherencia en la gestión de riesgos, la orientación a empresas mineras grandes, pequeñas y artesanales, y los enfoques holísticos de riesgo (Hermanus, 2007).

\section{Conclusiones}

Se identificó que los profesionales encargados de la administración y dirección de operaciones de estas empresas en su mayoría no tienen la especialidad competente en temas de Administración de Operaciones y Producción, no tienen conocimiento alguno de las posibles causas que están afectando a su industria. Existen canales débiles de comunicación entre la industria y la academia para la aplicación de los conocimientos adquiridos en formación, los cuales podrían solucionar problemas dentro de la industria y generar nuevos conocimientos científicos para el desarrollo del sector y la región.

La Administración total de la calidad identifica el desconocimiento de herramientas como control estadístico de procesos, lo que muestra la no identificación y control de variables que determinan la calidad del producto. Por otro lado, la poca utilización de diagramas de proceso, causa efecto para la identificación de causas a problemas, el no desarrollo de la función de calidad, evidenciando la poca caracterización de las necesidades del cliente para el desarro11 y diseño de sus productos. Así mismo, el lanzamiento de nuevos productos o productos mejorados no se considera, porque no invierten en investigación, innovación, diseño y desarrollo para el mejoramiento de los productos y otros elementos complementarios del proceso productivo. 
La Distribución en planta permite al momento de distribuir sus recursos, que no cuentan con definidas ni demarcadas áreas de la maquinaria, recepción de materia prima, almacenamiento de producto terminado, deficiencia en el diseño de instalaciones eléctricas, estructurales, agua e instalaciones sanitarias y una carencia en la distribución de los espacios para los recursos productivos, como también las condiciones de los componentes de la infraestructura física, en su diseño y construcción.

La Planificación de proyectos en las industrias evidencia no contar con políticas de capacitación y por consiguiente el personal no cuenta con las competencias de administración de proyectos. De la misma manera, el desconocimiento en la legislación minera, laboral y de seguridad y salud en el trabajo por parte de los empresarios del sector de transformación de material minero carbonatado, hacen que estos omitan y admitan sus deberes y derechos de estas normatividades.

La seguridad y salud en el trabajo, no es implementada, ni se le realiza un seguimiento adecuado, el personal trabaja en ambientes laborales que no cumplen los estándares de programas especializados de seguridad y salud en el trabajo debido a los diferentes riesgos laborales a que están expuestos tales como biomecánicas, físicos, locativos, mecánicos, químicos, psicosociales y la falta de medidas de intervención y control en la fuente, medio y trabajados en la labor de sus procesos productivos.

\section{Referencias bibliográficas}

Amponsah, K; Ofori, M. y Mensah, J. 2015. Occupational Health and Safety Management and Turnover Intention in the Ghanaian Mining Sector. Safety and Health at Work xxx 1-6.

Anand, G; Ward, P; Tatikonda, M. y Schilling, D. 2009. Dynamic capabilities through continuous improvement infrastructure, Journal of Operations Management, 27, 444-461.

Baptista, C. 2013. Interaction processes in long-term relationships in the metal mining industry: Longitudinal case studies of capital equipment buying. Industrial Marketing Management, 42(6), 969-982.

Bebbington, A; Bury, J; Cuba, N. \& Rogan, J. 2015. Mining, risk and climate resilience in the 'other' Pacific: Latin American lessons for the South Pacific. Asia Pacific Viewpoint, 56(2), 189-207.
Cárdenas, M. 2008. La minería en Colombia: impacto socioeconómico y fiscal. Colombia: Cuadernos de Fedesarrollo Número veinticinco, p. 153.

Chattopadhyay, S. \& D. Chattopadhyay. 2014. Mining Industries and Their Sustainable Management. En: Robert A. Meyers (ed.), Encyclopedia of Sustainability Science and Technology, pp. 6645-6665.

Delgado, M; Ulloa, C. \& Ramirez, J. 2015. La Economía del Departamento del Huila: Diagnóstico y Perspectivas de Mediano Plazo. Fedesarrollo. Centro de Investigacion Economica y Social, p. 84.

Fuente, M. E. 2013. Mineria Como Factor de Desarro1lo. Problemas del Desarrollo, pp. 177-198.

Gang, J; Tu, Y; Lev, B; Xu, J; Shen, W; \& Liming, Y. 2015. A multi-objective bi-level location planning problem for stone industrial parks. Computers \& Operations Research, 56, 8-21.

Gonzalez, M. B. 2008, Redifinicion De La Estructura Organizacional, Para la Empresa Calcalizas De Nobsa, Boyaca, Colombia. Global Conference on Business and Finance Proceedings, pp. 183-195.

Gowrisankaran, G; He, C; Lutz, E. y Burgess, J. 2015. Productivity, Safety, and Regulation in Coal Mining: Evidence from Disasters and Fatalities. NBER Working Paper No. 21129, p, 49.

Haslam, P. y Ary Tanimoune, N. 2016. The Determinants of Social Conflict in the Latin American Mining Sector: New Evidence with Quantitative Data. World Development, 78, 401-419.

Hermanus, M. 2007. Occupational health and safety in mining - Status, new developments, and concerns. Journal of the Southern African Institute of Mining and Metallurgy, 107(8), pp. 531-538.

Hung, Y; Huang, C; \& Ying, Y. 2013. Real-time capacity requirement planning for make-to-order manufacturing with variable time-window orders. Computers \& Industrial Engineering, 64(2), 641-652.

Jian, M; Fang, X; Jin, L. \& Azamat, R. 2015. The impact of lead time compression on demand forecasting risk and production cost: A newsvendor model. Transportation Research Part E: Logistics and Transportation Review, 84, 61-72. 
Olhager, J. \& Prajogo, D. 2012. The impact of manufacturing and supply chain improvement initiatives: A survey comparing make-to-order and make-tostock firms. Omega, 40(2), 159-165.

Robilliard, C. P. 2005. Las industrias extractivas y la aplicación de regalías a los productos mineros. Chile: CEPAL.

Sarmiento, Y. C. 2008. Restauracion en Explotacion de Minas Calizas. Luna Azul, 24-63.
Söderholm, K; Söderholm, P; Helenius, H; Pettersson, M; Viklund, R; Masloboev, V; Petrov, V. 2015. Environmental regulation and competitiveness in the mining industry: Permitting processes with special focus on Finland, Sweden and Russia. Resources Policy, 43, 130-142.

Tong, F. 2014. Simplificación Administrativa y «Regulación Positiva» en la Normativa Ambiental y Minera. Derecho \& Sociedad. Asociación Civil. 42, 279-287. 